\title{
Elaboração e validação do instrumento CICAc: classificação de idosos quanto à capacidade para o autocuidado*
}

\author{
Elaboration and validation of the CICAc \\ instrument: classification of the aged \\ concerning self care ability
}

Maria Helena Morgani de Almeida ${ }^{1}$

\begin{abstract}
ALMEIDA, M. H. M. Elaboração e validação do instrumento CICAc: classificação de idosos quanto à capacidade para o autocuidado. Rev. Ter. Ocup. Univ. São Paulo, São Paulo, v. 15, n. 3, p. 112-20, set./dez., 2004.

RESUMO: Através do presente artigo busca-se descrever o processo de elaboração e validação do Instrumento CICAc - Classificação de Idosos quanto à Capacidade para o Autocuidado - e apresentar os principais resultados obtidos. Propõe-se que seja utilizado por terapeutas ocupacionais junto a idosos especialmente em Unidades Básicas de Saúde (UBSs). O Instrumento foi elaborado em três etapas: Roteiro Preliminar, Roteiro Ajustado e Primeira Versão, através de análise de conteúdo de entrevistas com idosos $(n=24)$. Foi validado em seu conteúdo por terapeutas ocupacionais com especialidade em gerontologia $(n=15)$ através da Técnica Delphi. Para avaliar sua confiabilidade, foi aplicado duas vezes a idosos $(\mathrm{n}=30)$. Os itens do Instrumento obtiveram índices médios de aprovação pelos especialistas de 86,7\% para enunciado e $93,3 \%$ para conteúdo. Sua estabilidade, calculada através do coeficiente Kappa, foi avaliada de moderada à excelente. $\mathrm{O}$ instrumento apresentou consistência interna para os blocos das atividades básicas e instrumentais de vida diária com Alpha de Cronbach de 0,713 e 0,704 respectivamente. Conclui-se que o Instrumento CICAc, após os ajustes sofridos durante a validação, permite classificar idosos quanto à capacidade para o autocuidado e pode ser considerado apropriado à rotina em UBSs.
\end{abstract}

DESCRITORES: Avaliação. Idoso. Terapia Ocupacional. Atividades cotidianas. Autocuidado.

\footnotetext{
* Parte da Tese de Doutorado intitulada: "Validação do instrumento CICAc:classificação de idosos quanto à capacidade para o autocuidado", apresentada na FSP/USP

(1) Professora Doutora do Departamento de Fisioterapia, Fonoaudiologia e Terapia Ocupacional da FMUSP. Doutora em Saúde Pública pela FSP/USP.
}

Endereço para correspondência: Departamento de Fisioterapia, Fonoaudiologia e Terapia Ocupacional da FMUSP. Rua Cipotânea, 51. 05360-000. São Paulo, SP. E-mail: hmorgani@usp.br 


\section{INTRODUÇÃO}

$\mathrm{O}$ envelhecimento populacional é considerado um fenômeno mundial. O declínio es pecialmente das taxas de fecundidade tem provocado o envelhecimento da população brasileira de forma rápida desde a década de 1960 (CHAIMOWICZ, 1997).

Segundo o Censo do Ano 2000 (FUNDAÇÃO IBGE, 2000), a população de idosos no Brasil corresponde a 9,1\% ou cerca de 14.400 milhões de pessoas. De acordo com Kalache et al. (1987) a população brasileira crescerá cinco vezes até 2025 enquanto a população maior de 60 anos crescerá 15 vezes posicionando o Brasil em $6^{\circ}$ lugar no mundo em número absoluto de idosos .

Segundo Berquó (1996), a maior parte dos idosos brasileiros são mulheres, viúvas, morando sozinhas, na casa dos filhos ou chefiando famílias monoparentais, geralmente vivem em condições de pobreza pela baixa escolaridade e baixo rendimento econômico.

Estudos conduzidos no Brasil na década de 90 encontram ainda altas prevalências de incapacidades para atividades de vida diária, de $28 \%$ a $61 \%$ (LITVOC; DERNTL, 2002).

Segundo Litvak (1990), a susceptibilidade para enfermidades crônicas e incapacidades em idosos aumenta a demanda por serviços médicos e sociais, por políticas e programas que incluam medidas de prevenção de enfermidades crônicas e promoção da saúde.

\section{Promoção da saúde dos idosos e terapia ocupacional}

O paradigma da Promoção da saúde compreende saúde como "resultante de um conjunto de fatores sociais, econômicos, políticos, culturais, ambientais, comportamentais e biológicos" a ser equacionado por um modelo de atenção abrangente e explicativo e que supere, portanto, a abordagem clínico-assistencial (MINISTÉRIO DA SAÚDE, 1996, p. 12).

$\mathrm{Na}$ Carta de Ottawa, de acordo com o Ministério da Saúde (1996) Promoção da saúde é conceituada como “o processo de capacitação da comunidade para atuar na melhoria de sua qualidade de vida e saúde, incluindo uma maior participação no controle desse processo" (p. 12).

Segundo Restrepo (1994) a Promoção da saúde compreende medidas como educação para a saúde, promoção de políticas públicas, reorientação dos serviços e desenvolvimento de habilidades pessoais. É possível afirmar que a Promoção de saúde para idosos deva encaminhar-se para a estimação de um bom funcionamento mental, físico e social, e para a prevenção de enfermidades e incapacidades.
O princípio de fortalecimento da capacidade funcional compõe o paradigma da Promoção da saúde para idosos no Brasil (GORDILHO et al., 2000).

Segundo orientação do Ministério da Previdência e Assistência Social e Ministério da Saúde (1996) para o idoso manter-se em seu meio com a maior funcionalidade e autonomia possíveis deverá receber assistência interdisciplinar em todos os níveis de complexidade do Sistema Único de Saúde.

De acordo com a Secretaria Municipal da Saúde (1994) a possibilidade de atuação do Terapeuta Ocupacional (T.O.) se estende por toda a rede de recursos do Programa de Atenção a Saúde do Idoso, devendo este profissional compor a equipe interdisciplinar em todos os níveis de assistência a esta população.

Terapia ocupacional com idosos: ênfase na capacidade funcional

As perdas, inerentes ao processo de envelhecimento, afetam habitualmente o equilíbrio das atividades cotidianas. O T.O. atuará em antecipação ou na vigência de perdas, auxiliando o idoso a manter ou recobrar o equilíbrio de atividades em seu cotidiano.

Segundo Hasselkus (1998) a prática do T.O. em geriatria poderá dar ênfase à prevenção, recuperação ou adaptação. Quando enfoca a prevenção, dirige-se à prevenção de acidentes no domicílio e na comunidade, e à promoção de atividades.

Quando o idoso é portador de incapacidades, o T.O. poderá integrar a equipe interdisciplinar focalizando recuperação ou adaptação às incapacidades de acordo com o potencial de reversibilidade do quadro, devendo adotar como premissa, seja em programas com enfoque recuperativo ou adaptativo, o reforço das capacidades do idoso, de seus recursos próprios como motivações, experiências, movimentos e sentidos a serem complementados pela redução das demandas do ambiente, dispositivo auxiliar e/ou assistência quando necessário (ALMEIDA, 2003).

Muitos estudos reiteram a importância dessas estratégias para manter ou aumentar a independência para a vida cotidiana e as conceituam como práticas de autocuidado (NORBURN et al., 1995; ALMEIDA, 1997; MESSECAR, 2002; GITLIN et al., 2002).

Embora possibilidades para a atuação do T.O. com idosos tenham sido afirmadas em documento elaborado pela Secretaria Municipal da Saúde (1994) e estejam em consonância com dados de literatura, houve grande dificuldade para a materialização desta proposta, especialmente em Unidades Básicas de Saúde (UBSs). 
Através de estudo conduzido por Barros et al. (1995), sobre a incorporação da T.O. no contexto das ações em saúde mental e da pessoa portadora de deficiência no Município de São Paulo, foram identificados alguns fatores que dificultaram a sistematização da atenção pelo T.O. em UBSs, inclusive à idosos, como insuficiente experiência profissional com a diversidade da clientela, conhecimento limitado dos serviços em relação ao trabalho do profissional e precária disponibilidade de recursos para a área.

Com relação à população idosa, a dificuldade de sistematização da atenção deu-se ainda pela inexistência de consenso entre os T.O.s quanto ao instrumento de avaliação a ser adotado, levando-os a criarem ou adaptarem modelos de acordo com suas necessidades e concepções pessoais (ALMEIDA, 1997).

Admitindo-se que um instrumento específico de T.O. em gerontologia com possibilidade de uso consensual pudesse contribuir para sistematização da atenção profissional nesta área, especialmente em UBSs, foi elaborado e validado o Instrumento CICAc - Instrumento para a Classificação de Idosos quanto a Capacidade para o Autocuidado.

São propostas deste artigo descrever, de forma sucinta, o processo de elaboração e validação do Instrumento CICAc e apresentar os principais resultados obtidos.

Considerações sobre avaliação de terapia ocupacional em gerontologia: subsídios para a elaboração e validação do Instrumento CICAc

De acordo com Rogers (1996) para ser clinicamente eficiente a portadores de dificuldades de desempenho, a avaliação em T.O. deverá identificar atividades difíceis ou impossíveis para a pessoa realizar; porque ou quais as causas do problema; qual o potencial para reabilitação e como melhorar desempenho ou quais as melhores opções de intervenção.

Segundo Hasselkus (1998) o T.O. em geriatria deverá dar ênfase ao desempenho funcional enquanto comportamento resultante da interação entre a pessoa e o ambiente e, para tanto, adotar uma combinação de instrumentos para avaliar vários aspectos envolvidos no desempenho funcional de idosos como escalas de atividades de vida diária, instrumentos para avaliar áreas deficitárias, ambiente e recursos disponíveis.

Observa-se, no entanto que "a adoção de vários instrumentos de avaliação traz vantagens e desvantagens. Embora forneçam ampla gama de informações sobre o desempenho funcional do idoso, os conceitos que embasam esses instrumentos podem ser distintos e difi- cultarem a interpretação, integração e utilização das medidas obtidas. A pouca padronização no processo de aplicação destes instrumentos reduz a possibilidade de comparação de resultados, comprometendo especialmente sua utilização em estudos e pesquisas" (ALMEIDA, 2003, p. 37).

As informações sobre o universo ocupacional do idoso, além das atividades básicas e instrumentais de vida diária, devem também ser contempladas no processo de avaliação. De acordo com Horgas et al. (1998) o conhecimento sobre a diversidade das atividades do idoso provê informações sobre metas, habilidades, preferências, necessidades e desejos, restrições e oportunidades.

Acrescenta-se a importância ainda, no processo de avaliação, do reconhecimento de práticas de autocuidado para manter ou melhorar a funcionalidade na vida cotidiana. Elas indicam o potencial para a reabilitação e orientam sobre as opções para intervenção.

\section{Elaboração do instrumento CICAc}

O Instrumento CICAc foi elaborado por meio de estudo metodológico, composto por três etapas: Roteiro Preliminar, Roteiro Ajustado e Primeira Versão do Instrumento. O Roteiro Preliminar foi elaborado a partir de referenciais da Terapia Ocupacional, da área de Gerontologia e da teoria do Autocuidado. A aplicação do Roteiro Preliminar em entrevistas a idosos, usuários de uma UBS, seguida de análise temática e formal do conteúdo possibilitou a apreensão de informações relevantes sobre o autocuidado para estes idosos e a elaboração do Roteiro Ajustado. A aplicação desse roteiro em entrevistas aos idosos, não participantes da primeira etapa de entrevistas, seguida de análise do conteúdo destas entrevistas, gerou a Primeira Versão do Instrumento.

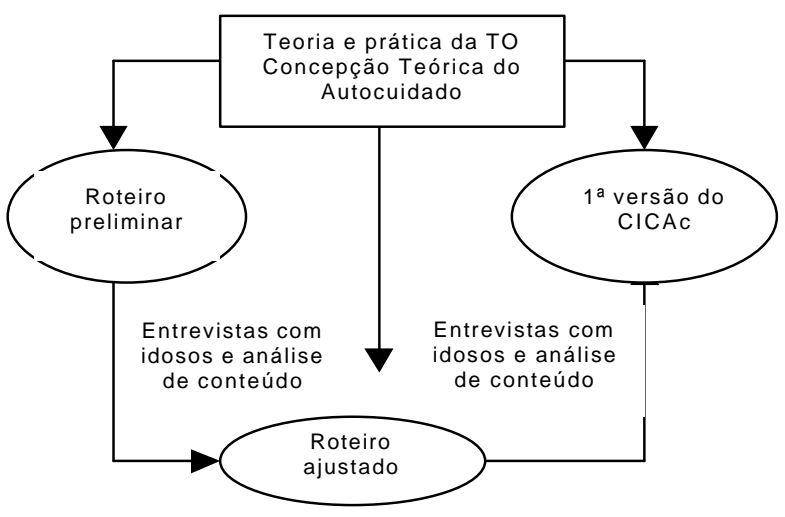

Fonte: Almeida, 1997.

Figura 1 - Etapas de elaboração da $1^{\text {a }}$ versão do instrumento CICAc. 


\section{População participante da elaboração do ins- trumento CICAc}

Foram selecionados 24 idosos para participar do estudo, com base nos seguintes critérios: ter se matriculado na UBS José Toledo Piza, no período de 02/01/ 95 a 15/04/95; possuir prontuário com informações sobre saúde e dados de identificação geral; mostrar disposição para participar do estudo e capacidade de compreensão e expressão de idéias.

\section{A primeira versão do instrumento CICAc}

Produto final do estudo metodológico em três etapas, a primeira versão do Instrumento CICAc é composta de variáveis e questões correspondentes, apre- sentadas de forma esquemática no Quadro 1.

Tabela 1 - Distribuição da população participante do estudo segundo sexo, faixa etária e participação das $1^{\mathrm{a}} \mathrm{e} 2^{\mathrm{a}}$ etapas de entrevistas.

\begin{tabular}{l|cc|cc|r}
\hline F.E/Sexo/Partic. & Entrev. Iniciais & \multicolumn{2}{|c|}{ Entrev. Compl. } & Total \\
& Fem. & Masc. & Fem. & Masc. & \\
\hline 60 a 69 anos & 3 & 1 & 3 & 2 & 9 \\
70 a 79 anos & 4 & - & 6 & - & 10 \\
80 anos ou mais & 2 & 1 & - & 2 & 5 \\
\hline Total & 9 & 2 & 9 & 4 & 24 \\
\hline
\end{tabular}

Fonte: Almeida M H M. CICAc: instrumento para classificação de idosos quanto à capacidade para o autocuidado. São Paulo; 1997. Dissertação (mestrado) - Faculdade de Saúde Pública da Universidade de São Paulo.

Quadro 1 - Variáveis da $1^{\text {a }}$ versão do CICAc e questões correspondentes.

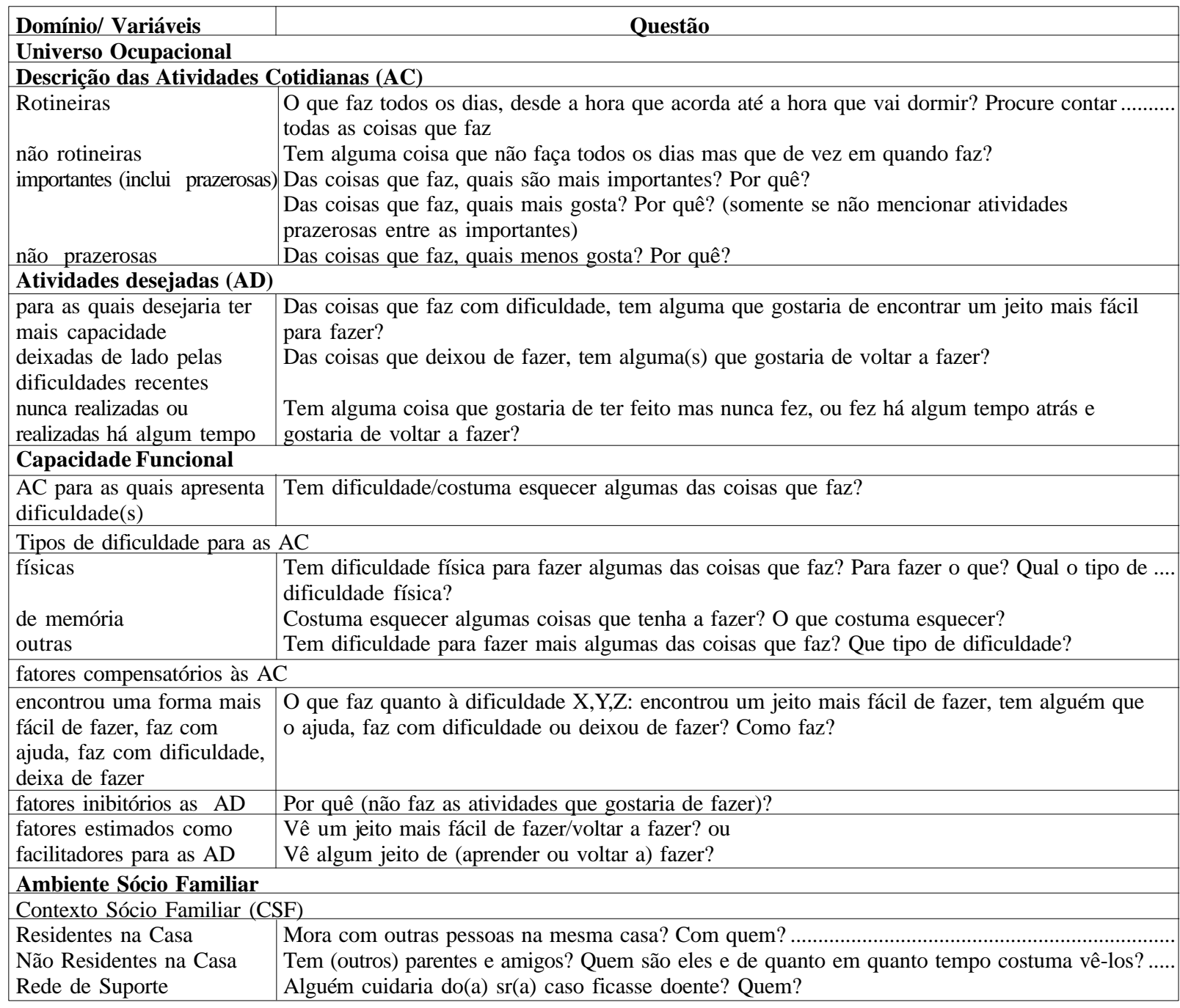

Fonte: Almeida, 1997. 


\section{Validação do instrumento CICAc}

O estudo de validação constou de duas etapas: validação de conteúdo e avaliação de estabilidade e consistência interna do Instrumento. Antes de submetêlo a essas estratégias, foi estruturado a partir do material gerado pela análise de conteúdo das entrevistas com idosos durante a fase de elaboração, ou seja, foram pré-estabelecidas opções de resposta para as questões do Instrumento. Segundo Almeida Filho (1989) instrumentos estruturados ajustam-se a algumas abordagens formais, permitem classificar indivíduos segundo características pesquisadas e podem oferecem resultados mais confiáveis.

\section{Validação de conteúdo}

Com o objetivo de obter uma amostra de itens relevantes para os distintos domínios de um instrumento, e a partir disso validar seu conteúdo, deve-se questionar pacientes, especialistas e consultar a literatura sobre aspectos que incluam propriedade, clareza e abrangência dos itens (McDOWELL; NEWELL, 1987; GIL, 1995). Optou-se, desta forma pelo emprego da Técnica Delphi para conduzir a validação de conteúdo do Instrumento CICAc, por se configurar como estratégia sistemática de ouvir e analisar opiniões de especialistas com possibilidade de gerar um produto validado (FARO, 1995).

$\mathrm{O}$ instrumento foi apreciado e ajustado por um júri de especialistas, T.Os $(\mathrm{n}=15)$ em três etapas. $\mathrm{Na}$ $\mathbf{1}^{\mathrm{a}}$ etapa foi solicitado aos juízes que opinassem individualmente sobre o Instrumento quanto às áreas, questões, formas de aplicação e possibilidade de conversão das escalas nominais em ordinais. $\mathrm{O}$ julgamento dos especialistas foi apreendido por meio de escalas de opinião, comentários e sugestões. Nas $2^{\mathrm{a}}$ e $3^{\mathrm{a}}$ etapas buscou-se obter o julgamento dos especialistas quanto ao conteúdo e enunciado das questões, sendo mantidos somente itens aprovados por $70 \%$ dos juízes ou mais.

\section{População participante do estudo de validação de conteúdo}

Foram selecionados e participaram efetivamente do estudo 15 T.Os com base nos seguintes critérios: atuação na área de gerontologia por um período igual ou superior a dois anos; residentes e atuantes no Estado de São Paulo; com interesse expresso em colaborar com o estudo

Destaca-se que 7 (47\%) especialistas contavam com 11 anos ou mais de atuação na área de gerontologia e $13(87 \%)$ possuíam curso de pós-graduação nesta área.

Síntese dos resultados obtidos no estudo de validação de conteúdo

Não houveram itens reprovados em seu conteúdo. Dois itens foram reprovados na $2^{\text {a }}$ etapa em seu enunciado, foram então reformulados, reapresentados na $3^{\text {a }}$ etapa e novamente julgados em seu conteúdo e enunciado quando então foram aprovados.

Gráfico 1 - Aprovação dos itens da $1^{\text {a }}$ versão grupal do Instrumento quanto ao enunciado. $2^{\text {a }}$ Etapa do Estudo Delphi, 2003.

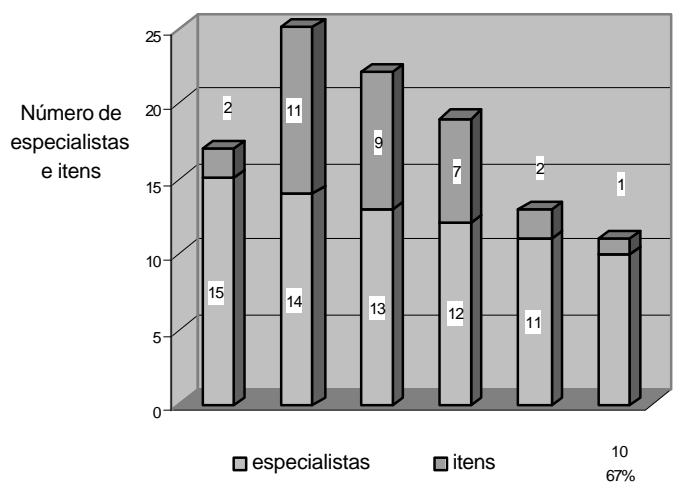

Fonte: Almeida, 2003.

$\mathrm{Na}$ etapa final do estudo, os índices médios de aprovação do Instrumento para conteúdo foram de $93,3 \%$ (com variação entre $80 \%$ e $100 \%$ ) e para enunciado foram de $86,7 \%$ (com variação entre $73,3 \%$ e $100,0 \%)$.

\section{Avaliação de estabilidade e consistência interna}

O Instrumento apreciado e ajustado pelos especialistas, em três etapas, culminou na $3^{\text {a }}$ versão grupal, esta versão foi aplicada duas vezes, com intervalo de 7 a 15 dias, a uma população de idosos $(\mathrm{n}=30)$. Antes da análise estatística foi necessário realizar agrupamentos de respostas para permitir a análise com um menor número de combinações de 
respostas possíveis (LIMA et al., 2003). Esta versão final foi denominada Versão simplificada do Instrumento CICAc, sua estabilidade foi calculada através do coeficiente Kappa e sua consistência interna foi calculada através do coeficiente Alpha de Cronbach.

\section{População participante da avaliação de esta- bilidade e consistência interna}

A $3^{\text {a }}$ versão grupal do Instrumento foi aplicada a 30 idosos, selecionados pelos seguintes critérios: ter 60 anos e mais; ser inscrito no Grupo de idosos do Centro de Saúde Escola Geraldo Paula Souza ou do Centro de Convivência Clube Chico Mendes ou ainda no Grupo Amizade, mantido pela Coordenação Regional de Obras da Promoção Humana; mostrar capacidade de compreensão e expressão de idéias; mostrar disposição e consentir em participar do estudo e; apresentar desempenho satisfatório no "Clock Completion Test".

Tabela 2 - População idosa participante da avaliação de confiabilidade do Instrumento CICAc segundo faixa etária e sexo. São Paulo, 2003.

\begin{tabular}{lccc}
\hline Faixa Etária / Sexo & Fem. & Masc. & Total \\
\hline 60 a 69 anos & 13 & - & 13 \\
70 a 79 anos & 10 & 1 & 11 \\
80 anos ou mais & 5 & 1 & 6 \\
\hline Total & 28 & 2 & 30 \\
\hline
\end{tabular}

\section{Síntese dos resultados obtidos na avaliação de estabilidade e consistência interna}

Para avaliação dos graus de concordância ou estabilidade, foi utilizado o critério de Stokes et al. (1995) citado por Lima et al. (2003), segundo o qual valores de Kappa abaixo de 0,4 indicam concordância fraca; entre 0,4 e 0,8 indicam concordância moderada e acima de 0,8 sugerem concordância excelente. Grande parte das medidas calculadas apresentou concordância de moderada a excelente, $60,14 \%$ foram maiores que 0,8 e apenas $7,17 \%$ foram menores que 0,4 .

O calculo da Alpha de Cronbach só foi possível para as escalas ordinais com um mesmo número de pontos e sem valores omissos, limitando o cálculo as áreas Capacidade Funcional e Perfil Social. O instrumento apresentou consistência interna para as atividades básicas e instrumentais de vida diária com Alpha de Cronbach de 0,713 e 0,704 respectivamente.

\section{Versão simplificada do instrumento CICAc}

Produto final do processo de validação, a Versão simplificada do Instrumento CICAc compreende: Orientações para aplicação, questões organizadas em domínios, objetivos correspondentes, quadros para registro de informações, instruções para preenchimento e duas linhas contínuas para posicionamento da capacidade funcional do idoso para os blocos das atividades básicas e instrumentais de vida diária ${ }^{1}$, totalizando sete páginas. O Quadro 2 apresenta a versão final do Instrumento CICAc de forma resumida com destaque para o conteúdo por ele contemplado.

\section{CONCLUSÕES}

O estudo Delphi gerou um instrumento válido em seu conteúdo, que possibilita o conhecimento de componentes relevantes do desempenho funcional do idoso. Embora forneça elementos importantes, a $3^{\text {a }}$ versão grupal do Instrumento demanda longo tempo de aplicação especialmente se considerada a rotina em UBSs. A Versão simplificada do Instrumento, organizada a partir do agrupamento de respostas, foi avaliada como confiável e pode ser considerada mais apropriada à rotina em UBSs e em pesquisas sobre envelhecimento por permitir a classificação sucinta de idosos quanto à capacidade para o autocuidado nas atividades cotidianas.

\footnotetext{
${ }^{1}$ A versão simplificada inclui escala para níveis de independência para as atividades cotidianas, construída pelos especialistas em sete níveis e reduzida a cinco níveis durante a análise de confiabilidade. Esta redução baseou-se em critérios teórico-metodológicos que buscaram assegurar sua exaustividade e especificidade. A versão simplificada permite atribuir pontuação ao idoso quanto à capacidade funcional para cada uma de suas atividades (de 1 a 5). Considerando-se que foi verificada a consistência interna do Instrumento para os blocos de atividades básicas e instrumentais de vida diária, ao idoso poderá atribuir-se ainda pontuação para o bloco das atividades básicas (de 9 a 45) e para o bloco das atividades instrumentais (de 8 a 40). Com base nesta pontuação, o idoso poderá ser posicionado numa "linha contínua" para cada um destes blocos.
} 
Quadro 2 - Questões, menção dos quadros para registro de respostas e instruções para preenchimento. Versão simplificada do Instrumento CICAc, 2003.

\begin{tabular}{|c|c|}
\hline Questão & Acompanha quadro para registro de... \\
\hline $\begin{array}{l}1 \text { - Mora com outras pessoas na mesma casa? Com quem? Identifique } \\
\text { todas as pessoas que moram com o idoso. }\end{array}$ & $\begin{array}{l}\text { nome, tipo de relacionamento, sexo e idade das } \\
\text { pessoas que moram com o idoso }\end{array}$ \\
\hline $\begin{array}{l}2 \text { - Tem (outros) filhos? Identifique todos os filhos vivos que não } \\
\text { moram com o idoso. }\end{array}$ & nome, sexo, idade e onde residem os filhos \\
\hline $\begin{array}{l}3 \text { - Com que freqüência se relaciona com: Preencha a coluna } \\
\text { correspondente à freqüência. }\end{array}$ & freqüências de relacionamentos com distintas pessoas \\
\hline $\begin{array}{l}4 \text { - Está satisfeito com a freqüência com se relaciona com: } \\
\text { assinale o grau de satisfação do idoso com a freqüência de cada } \\
\text { tipo de relacionamento. }\end{array}$ & $\begin{array}{l}\text { graus de satisfação do idoso quanto à freqüência com } \\
\text { que se relaciona com distintas pessoas }\end{array}$ \\
\hline $\begin{array}{l}5 \text { - Como se sente com a relação que mantém com: assinale o } \\
\text { grau de satisfação correspondente a cada um dos tipos de } \\
\text { relacionamento. }\end{array}$ & $\begin{array}{l}\text { graus de satisfação com relacionamentos com distintas } \\
\text { pessoas }\end{array}$ \\
\hline $\begin{array}{l}\text { 6 - Tem alguém que cuida do sr. quando fica doente? Quem? } \\
\text { Identifique as pessoas que cuidam do idoso em caso de doença. }\end{array}$ & $\begin{array}{l}\text { nome, tipo de relacionamento, sexo e idade daqueles } \\
\text { que cuidam do idoso }\end{array}$ \\
\hline $\begin{array}{l}\text { 7- Freqüentou escola? Até que ano estudou? Assinale a escolaridade } \\
\text { do idoso }\end{array}$ & grau de escolaridade do idoso \\
\hline 8 - Qual sua atividade profissional principal? & \multirow{3}{*}{$\begin{array}{l}- \\
-\end{array}$} \\
\hline $\begin{array}{l}9 \text { - Exerce essa atividade profissional atualmente? Se resposta } \\
\text { negativa ou dona de casa, aplique q. } 10\end{array}$ & \\
\hline $\mathbf{1 0}$ - Tem trabalho remunerado atualmente? & \\
\hline $\begin{array}{l}11 \text { - Quais são seus recursos financeiros atualmente? Assinale os } \\
\text { recursos financeiros na coluna à direita }\end{array}$ & recursos financeiros do idoso \\
\hline $\begin{array}{l}12 \text { - Com a sua situação econômica atual de que forma satisfaz suas } \\
\text { necessidades de alimentação, moradia, saúde, etc? Assinale grau de } \\
\text { satisfação de necessidades. }\end{array}$ & $\begin{array}{l}\text { grau de satisfação de necessidades com situação } \\
\text { econômica atual }\end{array}$ \\
\hline $\begin{array}{l}13 \text { - O que faz todos os dias, desde a hora acorda até a hora que vai } \\
\text { dormir? } \\
\mathbf{1 4} \text { - Tem alguma coisa que faça só de vez em quando? Assinale no } \\
\text { quadro } 1 \text { as atividades cotidianas do idoso. }\end{array}$ & $\begin{array}{l}\text { atividades que compõe o cotidiano do idoso (Quadro } 1 \\
\text { inclui "relação de atividades") }\end{array}$ \\
\hline $\begin{array}{l}15 \text { - Das coisas que faz, quais acha importantes? 15.1. Por quê? } \\
\text { Assinale no quadro } 1 \text { as atividades importantes e no quadro } 2 \\
\text { as razôes de importância atribuídas pelo idoso }\end{array}$ & $\begin{array}{l}\text { atividades importantes e razões de importância } \\
\text { (Quadro } 1 \text { para a q. } 15 \text { e Quadro } 2 \text { para a q. 15.1) }\end{array}$ \\
\hline $\begin{array}{l}16 \text { - Das coisas que faz, quais gosta menos? } 16.1 \text { Por quê? Assinale } \\
\text { no quadro } 1 \text { as atividades insatisfatórias e no quadro } 3 \text { as razões } \\
\text { atribuídas pelo idoso a sua insatisfação }\end{array}$ & $\begin{array}{l}\text { atividades insatisfatórias e razões de insatisfação } \\
\text { (Quadro } 1 \text { para a q.16 e Quadro } 3 \text { para a q.16.1) }\end{array}$ \\
\hline $\begin{array}{l}\mathbf{1 7} \text { - Tem dificuldade para fazer algumas das coisas que faz? Que } \\
\text { tipo(s) de dificuldade? Assinale no quadro } 4 \text { as atividades para quais } \\
\text { o idoso tem dificuldade e tipos de dificuldades } \\
\mathbf{1 8} \text { - O que faz quanto à dificuldade X: Encontrou um jeito mais fácil } \\
\text { de fazer, tem alguém que o(a) ajuda, faz mesmo com dificuldade sem } \\
\text { ajuda ou deixou de fazer? } \\
\mathbf{1 9} \text { - Caso tenha encontrado um jeito mais fácil de fazer, como faz? } \\
\text { Caso tenha alguém que o ajuda, quem o ajuda? Repita a pergunta } 18 \text { e } \\
19 \text { para cada dificuldade e assinale no quadro } 4 \text {, níveis de dificuldade } \\
\text { e formas de compensação }\end{array}$ & $\begin{array}{l}\text { de atividades para quais o idoso tem dificuldade, sua } \\
\text { caracterização segundo tipos de dificuldade } \\
\text { (biológica/comportamental/ do ambiente físico ou } \\
\text { social), níveis de dificuldade e formas de } \\
\text { compensação: Não apresenta dificuldade; } \\
\text { Apresenta dificuldade, mas compensa com uma ou } \\
\text { mais estratégias; Apresenta e faz com dificuldade; } \\
\text { Apresenta dificuldade e faz com ajuda; Apresenta } \\
\text { dificuldade e deixa de fazer. (Quadro 4) } \\
\text { A "relação de atividades" do Quadro } 1 \text { e repetida } \\
\text { agora no Quadro } 4\end{array}$ \\
\hline $\begin{array}{l}20 \text { - Pense nas coisas que gostaria de ter feito em sua vida mas nunca } \\
\text { fez ou fez há muito tempo atrás e gostaria de voltar a fazer. 20.1: Por } \\
\text { que não faz? 20.2: Imagina algum jeito de vir a fazê-las? } \\
\text { Registre no quadro } 5 \text { a atividade desejada, fatores de inibição e } \\
\text { formas imaginadas de realização }\end{array}$ & $\begin{array}{l}\text { de atividades desejadas, fatores de inibição e formas } \\
\text { imaginadas de realização (Quadro 5) }\end{array}$ \\
\hline
\end{tabular}

Fonte: Almeida, 2003. 
ALMEIDA, M. H. M. Elaboration and validation of the CICAc instrument: classification of the aged concerning self care ability. Rev. Ter. Ocup. Univ. São Paulo, São Paulo, v. 15, n. 3, p. 112-20, set./dez., 2004.

\begin{abstract}
The paper describes the elaboration and validation process of the CICAc Instrument - classification of the aged concerning self care ability - and to present the main results. It is considered that it is used by occupational therapist in regard to the aged, specially in Basic Health Units (BHU). The instrument was elaborated in three phases: Preliminary Questionnaire, Adapted Questionnaire, and First Version of the Instrument, from the interviews with elderly people and analysis of the content of these interviews. To establish the validity of the content, the instrument has been appraised and adjusted by a judging team of specialists, in three phases, employing the Delphi Technique. To assess its reliability it has been applied twice to a group of elderly people. The items researched have achieved average rates of approval of 93,3\% regarding content and $86,7 \%$ regarding statement. Its stability has been calculated using the Kappa coefficient, which showed moderate to excellent concordance. The instrument presented internal consistency concerning both basic and instrumental activities of daily living, the Alpha of Cronbach being 0,713 and 0,704 respectively. After the validation process, the CICAc Instrument allows classification of the elderly concerning self care ability and, it can be considered appropriate to the BHU routine.
\end{abstract}

KEY WORDS: Assessment. Aged. Occupational therapy. Activities daily living. Self care.

\title{
REFERÊNCIAS
}

ALMEIDA FILHO, N. Epidemiologia sem números: uma introdução crítica à ciência epidemiológica. Rio de Janeiro: Campos, 1989.

ALMEIDA, M. H. M. CICAc: instrumento para classificação de idosos quanto à capacidade para o autocuidado. 1997. 207 f. Dissertação (Mestrado) - Faculdade de Saúde Pública da Universidade de São Paulo, São Paulo, 1997.

ALMEIDA, M. H. M. Validação do instrumento CICAc: classificação de idosos quanto à capacidade para o autocuidado. 2003. 142 f. Tese (Doutorado) - Faculdade de Saúde Pública da Universidade de São Paulo, São Paulo, 2003.

BARROS, D. D.; OLIVER, F. C.; LOPES, R. E. Novas propostas assistenciais em São Paulo: estudo sobre a recente incorporação da terapia ocupacional no contexto das ações de saúde mental e saúde da pessoa portadora de deficiência física, sensorial ou mental no Município de São Paulo. São Paulo, 1995. [Relatório de pesquisa apresentado ao Conselho Nacional de Desenvolvimento Científico e Tecnológico].

BERQUÓ, E. Algumas considerações demográficas sobre o envelhecimento da população no Brasil. In: SEMINÁRIO INTERNACIONAL SOBRE ENVELHECIMENTO POPULACIONAL: uma agenda para o fim do século, 1996; Brasília, DF.
CHAIMOWICZ, F. A saúde dos idosos brasileiros às vésperas do século XXI: problemas, projeções e alternativas. Rev. Saúde Pública, v. 31, p. 184-200, 1997.

FARO, A. C. M. Técnica Delphi na validação das intervenções de enfermagem. Rev. Esc. Enferm. USP, v. 31, p. 259-273, 1997.

FUNDAÇÃO IBGE. Brasil em números: 2000. Rio de Janeiro, 2000. v. 8.

GIL, A. C. Métodos e técnicas em pesquisa social. São Paulo: Atlas, 1995.

GITLIN, L. N.; WINTER, L.; DENNIS, M. P.; CORCORAN, M.; SCHINFELD, S.; HAUCK, W. W. Strategies used by families to simplify tasks for individuals with Alzheimer's disease and related disorders. Gerontologist, v. 42, p. 61-69, 2002.

GORDILHO, A.; SERGIO, J.; SILVESTRE, J.; RAMOS, L. R.; FREIRE, M. P. A.; ESPINDOLA, A.; et al. Desafios a serem enfrentados no terceiro milênio, pelo setor saúde na atenção integral ao idoso. Rio de Janeiro: UnAti, 2000.

HASSELKUS, B. R. Discapacidad funcional en ancianos. In: HOPKINS, H. L.; SMITH, H. D. Terapia ocupacional de Willard \& Spackmann. 8a. ed. Madrid: Panamericana, 1998. p. 742-752. 
HORGAS, A. L.; WILMS, H. U.; BALTES, M. M. Daily life in very old age: everyday activities as expression of successful living. Gerontologist, v. 38, p. 556-568, 1998.

KALACHE, A.; VERAS, R. P.; RAMOS, R. L. O envelhecimento da população mundial: um desafio novo. Rev. Saúde Pública, v. 21, p. 200-210, 1987.

LIMA, A. C. P.; BARROSO, L. P.; IWAMIZU, P. S.; OKURA, R. I. S. Relatório de análise estatística sobre o projeto "Validação do Instrumento CICAc (Classificação de Idosos quanto à Capacidade para o Autocuidado)". São Paulo: IME-USP, 2003.

LITVAK, J. El envejecimiento de la población: un desafio que va más allá del año 2000. Bol. Oficina Sanit. Panam., v. 109, p. 2-5, 1990.

LITVOC, J.; DERNTL, A. M. Capacidade funcional do idoso: significado e aplicações. In: CIANCIARULLO, T. I.; GUALDA, D. M. R.; SILVA, G. T. R.; CUNHA, I. C. K. O. Saúde na família e na comunidade. São Paulo: Robe, 2002. p. 268-319.

McDOWELL, I.; NEWELL, C. Measuring health: a guide to rate scales and questionnaires. Oxford: Oxford University Press, 1987.

MESSECAR, D. C. Caregivers' ability to make environmental modifications. J. Gerontol. Nurs., v. 26, n. 12, p. 32-42, 2000

MINISTÉRIO DA PREVIDÊNCIA E ASSISTÊNCIA SOCIAL/MINISTÉRIO DA SAÚDE. Política nacional do idoso: perspectiva governamental. Brasília (DF), 1996.

MINISTÉRIO DA SAÚDE. FUNDAÇÃO OSWALDO CRUZ. Promoção da saúde. Brasília (DF), 1996.

NORBURN, J. E. K. I.; BERNARD, S. L.; KONRAD, T. R.; WOOMERT, A.; DEFRIESE, G. H.; et al. Self-care and assistance from others in coping with functional status limitations among a national sample of older adults. J. Gerontol. B. Psychol. Sci. Soc. Sci., v. 50, p. 101-109, 1995.

RESTREPO, H. E.; PEREZ, E. A. Promoción de la salud de los ancianos. In: PEREZ, E. A.; GALINSK, D.; MARTINEZ, A. R. S.; AYÉNDEZ, M. S. La atención de los ancianos: un desafio para los años noventa. Washington (DC): OMS/OPS, 1994. (POS - Publicación Cientifica, 546).

ROGERS, J. C.; HOLM, M. B.; STONE, R. G. Evaluation of daily living tasks: the home care advantage. Am. J. Occup. Ther., v. 51, p. 410-422, 1997.

SECRETARIA MUNICIPAL DE SAÚDE DE SÃO PAULO. Centro para a Organização da Atenção à Saúde. A inserção do profissional de terapia ocupacional no Programa de Atenção à Saúde do Idoso. São Paulo, 1994. 